Artigo Original

\title{
Consumo de oxigênio e índice de esforço percebido em diferentes ritmos de execução na hidroginástica
}

\author{
Cristine Lima Alberton \\ Claudia Renata Cardoso Rothmann \\ Stephanie Santana Pinto \\ Marcelo Coertjens \\ Luiz Fernando Martins Kruel \\ Universidade Federal do Rio Grande do Sul (UFRGS), Porto Alegre, RS, Brasil
}

\begin{abstract}
Resumo: $\mathrm{O}$ objetivo do estudo foi correlacionar o consumo de oxigênio $\left(\mathrm{VO}_{2}\right)$ e o percentual do $\mathrm{VO}_{2}$ máximo $\left(\% \mathrm{VO}_{2 \text { máx }}\right)$ com o índice de esforço percebido (IEP) e comparar essas variáveis entre exercícios de hidroginástica executados no meio aquático em diferentes ritmos de execução. Seis mulheres realizaram quatro sessões de testes, uma no meio terrestre (teste de esforço máximo) e três no meio aquático, cada uma com a execução de um exercício de hidroginástica: corrida estacionária, deslize lateral e chute frontal até $45^{\circ}$. Cada exercício aquático foi realizado em três ritmos de execução, 80, 100 e 120bpm, durante 6 min (intervalo de $30 \mathrm{~min}$ ). Para todas as variáveis, não foram observadas diferenças significativas entre os três exercícios, todavia, as mesmas apresentaram diferenças significativas entre os ritmos, indicando que com o aumento do ritmo, o esforço para executar os exercícios foi intensificado. Correlações significativas foram observadas entre $\mathrm{VO}_{2}$ e IEP e entre $\% \mathrm{VO}_{2 \text { máx }}$ e IEP. Logo, baseado nessas associações, sugere-se que a prescrição da intensidade dos exercícios analisados possa ser feita através do IEP durante aulas de hidroginástica.
\end{abstract}

Palavras-chave: Consumo de Oxigênio. Exercício. Imersão.

\section{Oxygen consumption and rate of perceived exertion at different rhythms in water aerobics}

\begin{abstract}
The purpose of the study was to correlate the oxygen consumption $\left(\mathrm{VO}_{2}\right)$ and the percentage of maximal $\mathrm{VO}_{2}\left(\% \mathrm{VO}_{2 \max }\right)$ with the rate of perceived exertion (RPE) and to compare these variable between water aerobic exercises performed in aquatic environment at different rhythms. Six women performed four test sessions, one on dry land environment (maximal effort test) and three in aquatic environment, each one with the performance of a water aerobic exercise: stationary running, jumping jacks and frontal kick up to $45^{\circ}$. Each aquatic exercise was performed at three rhythms of execution, 80, 100 and $120 \mathrm{bpm}$, during 6 min (interval of $30 \mathrm{~min}$ ). For all variables, no significant differences were observed between three exercises, however, significant differences were found between rhythms of execution for all of them, indicating that with the increase of the rhythm, the effort to perform the exercises was intensified. Significant correlations were observed between $\mathrm{VO}_{2}$ and $\mathrm{RPE}$, and between $\% \mathrm{VO}_{2 \max }$ and RPE. Therefore, based on this association it is suggested that the prescription of intensity of the exercises analyzed can be done by RPE during water aerobic classes.
\end{abstract}

Keywords: Oxygen Consumption. Exercise. Immersion.

\section{Introdução}

A prática da hidroginástica tem aumentado muito nos últimos anos. Este acréscimo está relacionado a seus benefícios cardiorrespiratórios aliados à redução dos danos articulares quando comparados aos exercícios terrestres. Dessa forma, tanto os adeptos tradicionais de atividades físicas como as pessoas que estão iniciando uma atividade (indivíduos com sobrepeso, gestantes, sedentários e idosos) podem participar desta modalidade de exercício (KRUEL, 2000).

$\mathrm{O}$ consumo de oxigênio $\left(\mathrm{VO}_{2}\right)$, entre as diversas ferramentas existentes para avaliar os indicadores de esforço fisiológico, é o principal parâmetro utilizado para quantificar a intensidade nas pesquisas da área das atividades aquáticas
(JOHNSON et al., 1977; CASSADY; NIELSEN, 1992; DARBY; YAEKLE, 2000; ALBERTON et. al.,

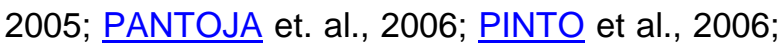
ALBERTON et. al., 2007; TIGGEMANN et. al., 2007; ALBERTON et. al., 2009). Todavia, na prática, utiliza-se amplamente a frequência cardíaca (FC) como ferramenta para a prescrição de exercícios aquáticos, uma vez que ela apresenta relação linear com $\circ \mathrm{VO}_{2}$ durante esforço ( $\underline{\text { HALL }}$ et al., 1998; $\underline{\mathrm{SHONO}}$ et al., 2000; ALBERTON et al., 2009), sendo de baixo custo e apresentando simplicidade na avaliação. Contudo, é importante salientar que em imersão a resposta da FC é atenuada devido aos efeitos hidrostáticos da água, fazendo-se necessário o uso de fórmulas de correção dos seus valores máximos 
ou realização de testes máximos aquáticos específicos para 0 seu uso adequado (ALBERTON; KRUEL, 2009). Portanto, é de fundamental importância 0 estudo do comportamento de outras variáveis que se associem com $\circ \mathrm{VO}_{2}$ e em adição, sejam de fácil aplicabilidade durante a prática da prescrição de exercícios aquáticos, tal como o índice de esforço percebido (IEP).

O IEP, mensurado através da Escala de Borg, tem recebido grande destaque na literatura como indicador de esforços aeróbicos gerais. Essa escala foi desenvolvida em 1961 com a intenção de ser uma ferramenta alternativa para a regulação e prescrição da intensidade do exercício (BORG, 1961). A sua principal característica é determinar o quão extenuante é um exercício, sendo que seus índices tem um aumento linear com o aumento da $\mathrm{FC}$ e do $\mathrm{VO}_{2}$
(BORG, 1982; DUNBAR et al. 1992). No meio aquático diversos estudos investigaram respostas fisiológicas e de IEP em diferentes exercícios de hidroginástica realizados em diferentes situações (BARBOSA et al., 2007; ALBERTON et al., 2008;

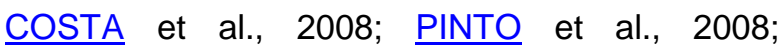
RAFAELLI et al., 2010; ALBERTON et al., 2011). No entanto, desses, apenas Alberton et al. (2011) analisaram a correlação entre o IEP e $0 \quad \mathrm{VO}_{2}$ $(r=0,6 ; p=0,001)$ em mulheres jovens executando um único exercício de hidroginástica, a corrida estacionária. Logo, evidencia-se a necessidade de melhor compreender as respostas do IEP e sua associação com $\circ \mathrm{VO}_{2}$ e percentual do $\mathrm{VO}_{2}$ máximo (\% $\left.\% \mathrm{~V}_{2 m a ́ x}\right)$ em diferentes exercícios de hidroginástica. Portanto, o objetivo deste estudo é correlacionar $0 \mathrm{VO}_{2}$ e $0 \% \mathrm{VO}_{2 \text { máx }}$ com o IEP e comparar essas variáveis entre três exercícios de hidroginástica executados no meio aquático em três diferentes ritmos de execução.

\section{Amostra}

\section{Materiais e Métodos}

A amostra do presente estudo foi composta por seis mulheres jovens voluntárias com idade entre 21 e 23 anos, cujas características são apresentadas na Tabela 1. Como critérios de inclusão, as mesmas deveriam ser aparentemente saudáveis, ativas, ambientadas ao meio líquido, praticantes de hidroginástica por no mínimo três meses e isentas de doenças músculoesqueléticas, ósteoarticulares, cardíacas, pulmonares ou uso de medicação. A seleção da amostra foi por conveniência, realizada através de convite verbal para as alunas do Centro Natatório da Escola de Educação Física de Universidade Federal do Rio Grande do Sul.

Tabela 1. Caracterização da Amostra - Média e desvio padrão (DP) da idade, massa corporal, estatura e consumo máximo de oxigênio $\left(\mathrm{VO}_{2 \text { máx }}\right)$.

\begin{tabular}{lcc}
\hline $\mathbf{N}=\mathbf{6}$ & Média & DP \\
\hline Idade (anos) & 21,30 & $\pm 1,03$ \\
Massa corporal $(\mathrm{kg})$ & 57,00 & $\pm 4,29$ \\
Estatura $(\mathrm{cm})$ & 164,50 & $\pm 4,09$ \\
$\mathrm{VO}_{\text {2máx }}\left(\mathrm{ml} \cdot \mathrm{kg}^{-1} \cdot \mathrm{min}^{-1}\right)$ & 44,20 & $\pm 2,27$ \\
\hline
\end{tabular}

\section{Procedimentos}

Uma sessão inicial foi realizada no Laboratório de Pesquisa da Universidade Federal do Rio Grande do Sul (UFRGS). Inicialmente, todos os sujeitos leram e assinaram um termo de consentimento informado contendo todas as informações pertinentes ao estudo. Após, foram obtidas as medidas de massa corporal e estatura, em uma balança de alavanca, com estadiômetro acoplado (FILIZOLA). A seguir, foi realizado um teste máximo na esteira ergométrica (modelo 10200 ATL, INBRAMED) com a finalidade de avaliar $\mathrm{o} \mathrm{VO}_{2}$ máximo $\left(\mathrm{VO}_{2 \text { máx }}\right)$. $\mathrm{O} \quad \mathrm{VO}_{2 \text { máx }}$ foi utilizado para a caracterização da amostra, assim como para a normalização dos valores de $\mathrm{VO}_{2}$ dos exercícios expressos como \% $\mathrm{VO}_{2 \text { máx. }} \mathrm{O}$ protocolo consistiu de uma velocidade inicial de 5 $\mathrm{km} \cdot \mathrm{h}^{-1} \mathrm{com} 1 \%$ de inclinação durante 2 minutos. Posteriormente, foram realizados incrementos de $1 \mathrm{~km} \cdot \mathrm{h}^{-1}$ na velocidade a cada minuto, com a manutenção da inclinação, até que os indivíduos atingissem o máximo esforço. Para garantir que cada sujeito tivesse alcançado o esforço máximo, pelo menos dois dos quatro critérios seguintes foram exigidos para a validação do teste: 1) um platô no $\mathrm{VO}_{2}$ com o aumento da intensidade do exercício; 2) uma razão de troca respiratória mínima de 1,15; 3) uma taxa respiratória máxima de no mínimo 35 respirações por minuto e 4) IEP 
mínimo correspondente ao valor 18 da escala de Borg (HOWLEY et al., 1995).

Os sujeitos participaram de uma aula inicial de hidroginástica para familiarizarem-se com os exercícios e com os índices da escala RPE de Borg de esforço percebido (BORG, 2000). Inicialmente, todas as instruções sobre a escala $\mathrm{RPE}$ foram ensinadas às participantes de acordo com as recomendações de Borg (2000). Posteriormente, cada exercício aquático foi realizado pelas participantes em todos os níveis de esforço, de 6 (extremamente fácil) a 20 (esforço máximo), para familiarizá-las com o mínimo de esforço e sua graduação até o esforço máximo, de acordo com sua percepção.
Posteriormente, três sessões de coletas foram realizadas no Centro Natatório da Escola de Educação Física da UFRGS, cada uma correspondente a um exercício específico de hidroginástica, a corrida estacionária (COR), o deslize lateral (DL) e o chute frontal até 45 $(\mathrm{CHU})$ (Figura 1), realizados com intervalos de 48 horas. Os seguintes critérios foram estabelecidos para as coletas de dados seguintes: alimentar-se no período de 3 a 4 horas antes do início da sessão de testes, sem a ingestão de estimulantes e evitar a prática de atividades físicas intensas durante as últimas 24 horas (OOKE, 1996).

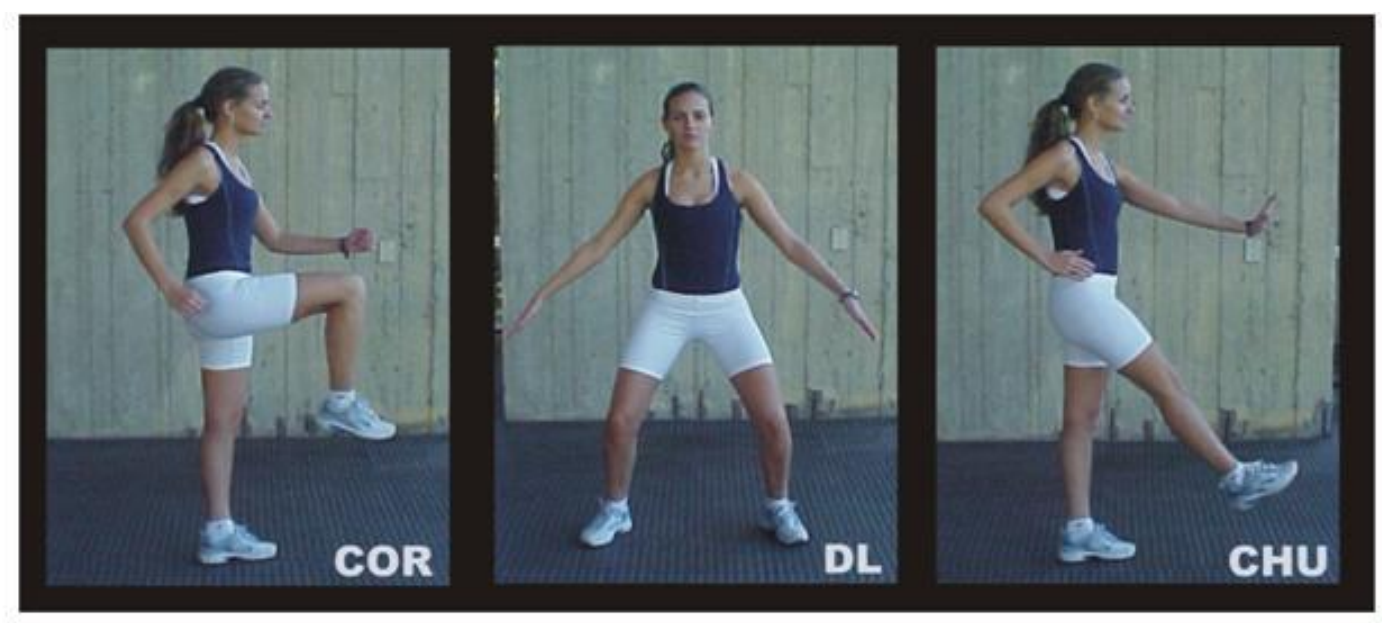

Figura1. Exercícios realizados: (COR) Corrida estacionária. (DL) Deslize lateral. (CHU) Chute frontal a 45ํ․

Em cada sessão, o sujeito permanecia inicialmente em repouso na posição de decúbito dorsal fora da água durante 30 minutos. Nos últimos 10 minutos de repouso os valores de $\mathrm{VO}_{2}$ eram registrados e analisados a fim de certificar que todos os sujeitos iniciavam o experimento com o mesmo estado metabólico de repouso. A seguir, os indivíduos entravam na piscina para a coleta em exercício nas diferentes cadências. O exercício foi realizado randomicamente nos ritmos de execução de 80, 100 e 120 bpm, cada um com duração de 6 minutos, respeitando 30 minutos de intervalo. Os diferentes ritmos de execução foram controlados e reproduzidos por um metrônomo (MATRIX MR-500, marca Quartz). Em todas as sessões, os exercícios foram executados em imersão na profundidade do processo xifoide, com temperatura da água mantida entre 32 e $33^{\circ} \mathrm{C}$.

Para a avaliação do consumo de oxigênio foi utilizado um analisador de gases portátil, do tipo caixa de mistura (KB1-C, marca AEROSPORT, Ann Arbor, USA). Previamente ao início dos testes, o equipamento foi calibrado através de gases com concentrações conhecidas e foi realizada uma calibração de volume para cada faixa de volume medida. Entre cada teste foi realizada uma calibração automática com base nos valores de volume e gases ambientes. Utilizou-se um pneumotacógrafo de fluxo baixo ( 2 a $301 . \mathrm{min}^{-1}$ ) para as coletas em repouso e de fluxo médio (10 a $120 \mathrm{l} . \mathrm{min}^{-1}$ ) para as coletas em exercício. $\mathrm{O} \mathrm{VO}_{2}$ foi coletado com uma frequência de uma amostra a cada 20 segundos nos últimos 3 minutos de cada situação, tanto em repouso como no exercício. Realizou-se uma média dos 3 minutos coletados em cada situação. Além disso, os valores de $\% \mathrm{VO}_{2 m a ́ x}$ foram calculados e normalizados a partir dos valores de $\mathrm{VO}_{2 m a ́ x}$ e $\mathrm{VO}_{2}$ em cada situação obtidos de cada indivíduo. Para coletar o IEP, a Escala RPE de Borg foi utilizada (BORG, 2000). De acordo com Borg (2000), sua 
escala possui 14 pontos, partindo de 6 até 20 . 0 IEP foi coletado imediatamente ao final de cada cadência de execução.

\section{Análise Estatística}

Para analisar os dados coletados, foi utilizada estatística descritiva, com os dados apresentados através de médias \pm desvio-padrão (DP). Utilizouse o teste de normalidade de Shapiro-Wilk para a análise da distribuição dos dados e os resultados justificam o uso de estatística paramétrica. ANOVA para medidas repetidas foi utilizada para comparar o $\mathrm{VO}_{2}$ na situação de repouso entre os três dias de coleta. Para a análise do $\mathrm{VO}_{2}$, do $\% \mathrm{VO}_{2 \text { máx }}$ e do IEP durante o exercício utilizou-se ANOVA de dois fatores para medidas repetidas (ritmos de execução e exercício). Para a localização das diferenças significativas entre as situações utilizou-se o teste complementar de Bonferroni. O teste de Correlação Linear de Pearson foi utilizado a fim de verificar o nível de associação entre as variáveis analisadas. $O$ índice de significância adotado neste estudo foi de $\alpha=0,05$. O tratamento estatístico foi realizado no pacote estatístico SPSS versão 13.0.

\section{Resultados}

A Tabela 2 apresenta as respostas do $\mathrm{VO}_{2}$ na situação de repouso pré-exercício durante os três dias de coleta. Os resultados da análise $(p=0,187)$ demonstraram não haver diferenças significativas entre os três dias de experimento. Logo, pode-se inferir que os indivíduos partiram do mesmo estado metabólico de consumo de oxigênio de repouso e, portanto, os aumentos nesta variável durante 0 experimento foram causados pelo esforço exigido nas atividades deste protocolo.

Tabela 2. Médias, desvio padrão (DP) e significância do consumo de oxigênio $\left(\mathrm{VO}_{2}\right)$ de repouso pré-execução do exercício nos três dias de coleta.

\begin{tabular}{|c|c|c|c|}
\hline \multirow{2}{*}{ Dia } & \multicolumn{2}{|c|}{$\mathrm{VO}_{2}\left(\mathrm{ml} \cdot \mathrm{kg}^{-1} \cdot \mathrm{min}^{-1}\right)$} & \multirow{2}{*}{ Sig. } \\
\hline & Média & DP & \\
\hline 1 & 3,51 & $\pm 0,45$ & \\
\hline 2 & 3,21 & $\pm 0,54$ & 0,187 \\
\hline 3 & 3,14 &, \pm 033 & \\
\hline
\end{tabular}

Os resultados descritivos do $\mathrm{VO}_{2}, \% \mathrm{VO}_{2 \text { máx }} \mathrm{e}$ do IEP nas diferentes situações de exercício estão apresentados na Tabela 3. De acordo com a ANOVA de dois fatores para medidas repetidas, não houve efeito significativo do fator exercício $\left(\mathrm{VO}_{2}: \mathrm{p}=0,694 ; \% \mathrm{VO}_{2 \text { máx }} \mathrm{p}=0,548\right.$; IEP: $\left.\mathrm{p}=0,073\right)$, indicando que independente do ritmo de execução o comportamento das variáveis foi semelhante entre os exercícios. No entanto, para todas as variáveis 0 efeito ritmo de execução foi significativo $\left(\mathrm{VO}_{2}: \mathrm{p}<0,001 ; \% \mathrm{VO}_{2 \text { máx: }} \mathrm{p}=0,02\right.$; IEP: $p<0,001)$, com diferenças significativas entre o ritmo de $120 \mathrm{bpm}$ e as intensidades mais baixas para $\circ \mathrm{VO}_{2}$ e entre todas as intensidades para $\% \mathrm{VO}_{2 \text { máx }}$ e IEP, conforme demonstrado na Figura 2. Além disso, não houve interação significativa para nenhuma das variáveis analisadas $\left(\mathrm{VO}_{2}\right.$ : $\mathrm{p}=0,092 ; \% \mathrm{VO}_{2 m a ́ x}: \mathrm{p}=0,310$; IEP: $\left.\mathrm{p}=0,225\right)$.

Tabela 3. Média, desvio padrão (DP) e níveis de significância dos efeitos principais exercício e ritmo de execução e interação exercício*ritmo para as variáveis consumo de oxigênio $\left(\mathrm{VO}_{2}\right)$, percentual do consumo de oxigênio máximo $\left(\% \mathrm{VO}_{2 m a ́ x}\right)$ e do índice de esforço percebido (IEP) nos exercícios corrida estacionária (COR), chute frontal até $45^{\circ}(\mathrm{CHU})$ e deslize lateral (DL) executados nas diferentes cadências.

\begin{tabular}{|c|c|c|c|c|c|c|c|c|c|c|}
\hline \multirow{3}{*}{ Variável } & \multirow{3}{*}{ Ritmo } & \multicolumn{6}{|c|}{ Exercício } & \multirow{3}{*}{$\begin{array}{c}\text { Exercício } \\
\text { Sig. }\end{array}$} & \multirow{3}{*}{$\begin{array}{c}\text { Ritmo } \\
\text { Sig. }\end{array}$} & \multirow{3}{*}{$\begin{array}{c}\begin{array}{c}\text { Exercício } \\
\text { *Ritmo }\end{array} \\
\text { Sig. }\end{array}$} \\
\hline & & \multicolumn{2}{|c|}{ COR } & \multicolumn{2}{|c|}{$\mathrm{CHU}$} & \multicolumn{2}{|c|}{$\mathrm{DL}$} & & & \\
\hline & & Média & DP & Média & DP & Média & DP & & & \\
\hline \multirow{3}{*}{$\begin{array}{c}\mathrm{VO}_{2} \\
\left(\mathrm{ml}^{-k^{-1}} \cdot{ }^{-1}\right)\end{array}$} & 80bpm & 13,01 & $\pm 2,36$ & 13,56 & $\pm 4,41$ & 11,53 & $\pm 2,56$ & \multirow{3}{*}{0,694} & \multirow{3}{*}{$\stackrel{<}{<.001}$} & \multirow{3}{*}{0,092} \\
\hline & 100bpm & 14,38 & $\pm 2,08$ & 14,07 & $\pm 2,42$ & 14,73 & $\pm 2,58$ & & & \\
\hline & 120bpm & 20,25 & $\pm 4,82$ & 18,20 & $\pm 4,32$ & 17,76 & $\pm 4,82$ & & & \\
\hline \multirow{3}{*}{$\begin{array}{c}\% \mathrm{VO}_{2 \text { máx }} \\
(\%)\end{array}$} & 80bpm & 29,32 & $\pm 4,34$ & 26,92 & $\pm 2,50$ & 25,93 & $\pm 4,74$ & \multirow{3}{*}{0,548} & \multirow{3}{*}{0,02} & \multirow{3}{*}{0,310} \\
\hline & 100bpm & 32,50 & $\pm 4,18$ & 31,89 & $\pm 5,78$ & 33,28 & $\pm 5,41$ & & & \\
\hline & 120bpm & 45,56 & $\pm 9,05$ & 41,25 & $\pm 10,10$ & 40,80 & $\pm 9,11$ & & & \\
\hline \multirow{3}{*}{ IEP } & $80 \mathrm{bpm}$ & 9,83 & $\pm 0,98$ & 9,33 & $\pm 0,82$ & 10,00 & $\pm 1,10$ & \multirow{3}{*}{0,073} & \multirow{3}{*}{$\stackrel{<}{<, 001}$} & \multirow{3}{*}{0,225} \\
\hline & 100bpm & 11,83 & $\pm 0,75$ & 11,50 & $\pm 0,55$ & 12,67 & $\pm 1,17$ & & & \\
\hline & 120bpm & 13,67 & $\pm 0,82$ & 13,33 & $\pm 1,03$ & 15,33 & $\pm 1,86$ & & & \\
\hline
\end{tabular}



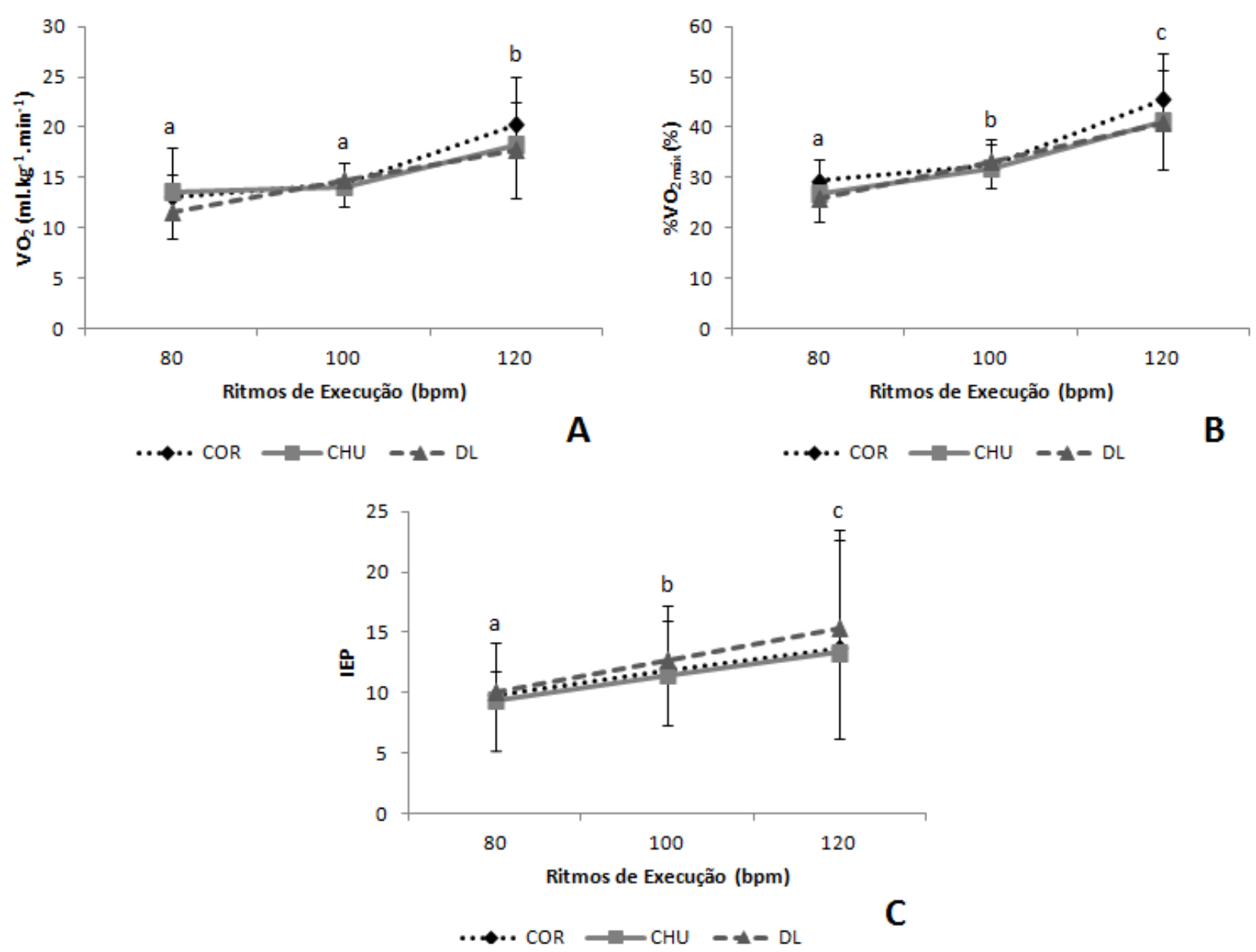

Figura 2. Resultados do consumo de oxigênio $\left(\mathrm{VO}_{2}\right)(\mathrm{A})$, do percentual do consumo de oxigênio máximo $\left(\mathrm{VO}_{2 \text { máx. }}\right.$ (B), e do índice de esforço percebido (IEP) (C) para exercícios corrida, deslize lateral e chute frontal realizados nos diferentes ritmos de execução. Nota: Letras diferentes indicam diferença estatisticamente significativa entre os ritmos de execução.

Uma vez que os exercícios não apresentaram diferenças significativas, todos foram agrupados para a análise de correlação. Os resultados das correlações entre $\mathrm{VO}_{2}$ e IEP $(r=0,652 ; p<0,001)$ e entre $\% \mathrm{VO}_{2 m a ́ x} \mathrm{e}$ IEP ( $r=0,679 ; p<0,001)$ estão demonstrados na Figura 3. De acordo com a proposta de Calegari-Jacques (2004), esses valores de correlação podem ser considerados fortes e significativos, indicando associação entre as variáveis fisiológicas e o IEP.
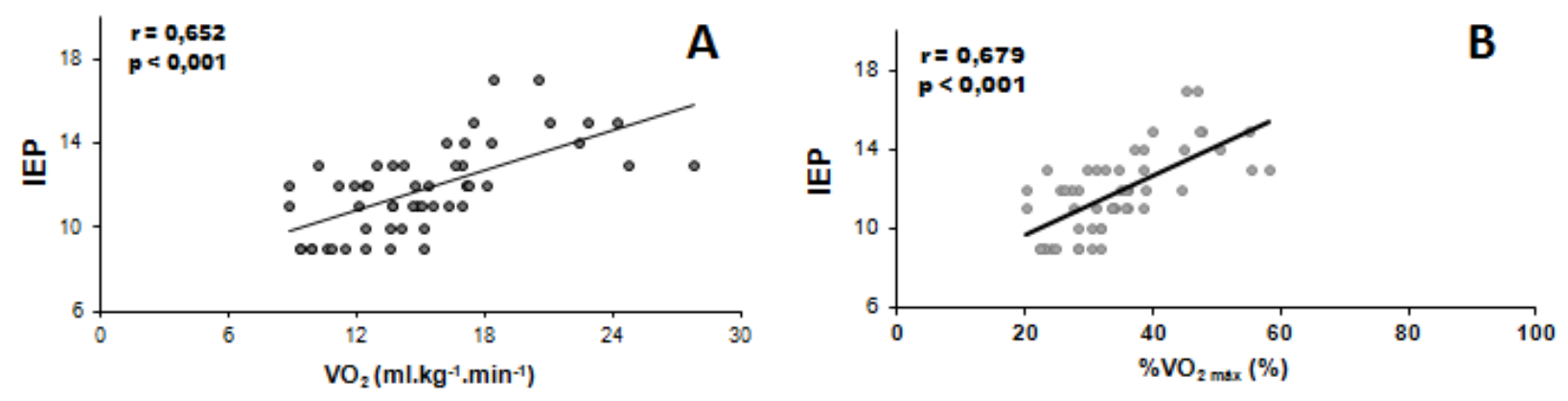

Figura 3. Correlações entre consumo de oxigênio $\left(\mathrm{VO}_{2}\right)$ e índice de esforço percebido (IEP) (A) e entre percentual do consumo de oxigênio máximo de $\left(\% \mathrm{VO}_{2 \text { máx. }}\right)$ e índice de esforço percebido (B). 


\section{Discussão}

No presente estudo foram encontradas correlações significativas entre $\circ \mathrm{VO}_{2}$ e $\circ \mathrm{IEP}$, assim como entre $0 \% \mathrm{VO}_{2 \text { máx }}$ e o IEP. Também foi possível verificar que, com o aumento do ritmo de execução, o IEP e $\quad \circ \quad \mathrm{VO}_{2}$ aumentaram significativamente independente do exercício executado.

Há diversos fatores que influenciam a demanda energética de uma atividade. Em exercícios aquáticos a área de superfície projetada, a velocidade de execução em relação ao meio e a resistência por ele exercida são fatores determinantes do esforço ( al., 1977; ALBERTON et al., 2005).

Quando exercícios diferentes são executados em um ritmo de execução fixo, os segmentos corporais nem sempre produzem esforços semelhantes. Os estudos de JOHNSON et al. (1977), CASSADY; NIELSEN (1992), PINTO et al. (2006) e ALBERTON et al. (2008) compararam as respostas cardiorrespiratórias $\left(\mathrm{VO}_{2}\right.$ e $\left.\mathrm{FC}\right)$ de dois diferentes exercícios executados num mesmo ritmo de execução e observaram diferenças significativas entre eles. Tais resultados são devidos às diferentes amplitudes dos segmentos utilizados em cada exercício. Além disso, as massas musculares envolvidas e a velocidade angular de cada segmento também são dependentes do padrão de movimento no exercício realizado. Essas variações produzem diferentes resistências do meio em relação ao próprio segmento envolvido, resultando em esforços diferenciados para cada exercício.

Contudo, no presente estudo foram verificadas intensidades semelhantes de $\mathrm{VO}_{2}, \% \mathrm{VO}_{2 \text { máx }}$ e IEP para os três exercícios analisados em cada ritmo de execução. Tal resultado pode, provavelmente, ser explicado pelas diferenças nas áreas projetadas de cada segmento corporal em cada exercício analisado terem sido compensadas pelas diferenças nas massas musculares envolvidas (membros inferiores e membros superiores) e também na diferença na velocidade de cada segmento em relação ao meio. Esses achados corroboram o estudo de Alberton et al. (2007) que investigou o $\mathrm{VO}_{2}$ de oito diferentes exercícios de hidroginástica e encontrou grupos de exercícios de mesma intensidade dentro do mesmo ritmo de execução.

Por outro lado, conforme os resultados do presente estudo, os valores de IEP, de $\mathrm{VO}_{2}$ e de $\% \mathrm{VO}_{2 \text { máx }}$ aumentaram com o incremento do ritmo de execução. Esses dados corroboram outros estudos que também encontraram maiores respostas cardiorrespiratórias com o aumento do ritmo de execução em exercícios de hidroginástica (CASSADY; NIELSEN, 1992; ALBERTON et al., 2005; ALBERTON et al., 2009) ou caminhada aquática (GLEIM; NICHOLAS,

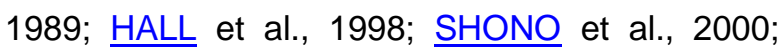
POHL; McNAUGHTON, 2003). Em estudo realizado em esteira aquática, Shono et al. (2000) analisaram diversas variáveis, dentre elas $\circ \mathrm{VO}_{2}$, a FC e o IEP, durante a caminhada executada em quatro velocidades, e concluíram que o IEP aumentou com o aumento da velocidade de execução, assim como as demais variáveis.

Essas respostas ocorrem devido ao aumento da velocidade do corpo em relação ao fluído, provocando um grande aumento na resistência. Essa maior resistência ao avanço pode ser explicada pelo fato de a velocidade ser elevada ao quadrado e diretamente proporcional a ela (ALEXANDER, 1977). Assim, quanto maior a velocidade de execução de um determinado exercício no meio aquático, maior é a resistência oferecida por esse fluído e, consequentemente, maior é a demanda necessária de oxigênio para a musculatura exercitada.

Ao analisar a associação entre $\mathrm{VO}_{2}$ e IEP, o presente estudo observou correlações significativas entre $\% \mathrm{VO}_{2 \text { máx }}$ e IEP $(r=0,679$; $p<0,001)$ e entre $\mathrm{VO}_{2}$ e IEP $(r=0,652 ; p<0,001)$ para a análise dos três exercícios de hidroginástica investigados. Tais valores concordam com as achados de Svedenhag; Seger, (1992) que observaram uma relação linear entre variáveis fisiológicas e o IEP na modalidade aquática de corrida em piscina funda. Da mesma forma, Shono et al. (2000) observaram uma correlação forte e significativa $(r=0,996 ; p<0,01)$ entre a FC e o IEP na caminhada em esteira aquática. Na hidroginástica, Alberton et al. (2011) também encontraram correlações significativas entre IEP e $\mathrm{VO}_{2}(r=0,6 ; p=0,001)$ e entre IEP e 
$\% \mathrm{VO}_{2 \text { máx }}(r=0,71 ; p<0,001)$, no entanto apenas um exercício, a corrida estacionária, foi analisado.

Tais linearidades podem ser provavelmente explicadas pela capacidade do sistema sensorial e proprioceptivo dos indivíduos de gerar uma percepção de esforço proporcional à demanda real de $\mathrm{VO}_{2}$ tanto absoluto como relativo ao $\mathrm{VO}_{2 \text { máx. }}$ De acordo com Borg (1961), ao realizar uma tarefa muscular intensa, recebemos sensações dos músculos, das articulações e dos receptores somatossensitivos, dos sistemas cardiovascular e respiratório e de outros órgãos do corpo. Com o aumento da demanda de $\mathrm{VO}_{2}$ que 0 corpo necessita, diversas outras adaptações biológicas podem ocorrer simultaneamente durante o exercício, tais como o aumento da FC, da pressão arterial sistêmica, do gasto energético e da concentração de lactato plasmático. Provavelmente, para os exercícios aquáticos analisados, o aumento da demanda de $\mathrm{VO}_{2}$ aliado ao acréscimo das outras adaptações citadas acima provoquem no indivíduo a sensação subjetiva de esforço proporcional ao quão extenuante é a atividade.

\section{Conclusão}

A partir da análise de dados do presente estudo, conclui-se que os exercícios analisados, chute frontal até $45^{\circ}$, corrida estacionária e deslize lateral, quando executados nos ritmos de execução fixas produzem esforços semelhantes e que esse esforço é intensificado com o aumento do ritmo de execução. Além disso, baseado nas correlações significativas observadas entre $\mathrm{VO}_{2} \mathrm{e}$ IEP e entre $\% \mathrm{VO}_{2 \text { máx }}$ e IEP, sugere-se que a prescrição da intensidade dos exercícios analisados possa ser feita através do IEP durante aulas de hidroginástica.

\section{Referências}

ALBERTON, C. L.; COERTJENS, M.;

FIGUEIREDO, P. A. P.; KRUEL, L. F. M. Behavior of oxygen uptake in water exercises performed at different cadences in and out of water. Medicine and Science in Sports and Exercise, Madison, v. 37, n. 5, p. S103, 2005.

ALBERTON, C. L.; OLKOSKI, M. M.; BECKER, M. E.; PINTO, S. S.; KRUEL, L. F. M. Cardiorespiratory responses of postmenopausal women to different water exercises. International Journal of Aquatic Research and Education, Bowling Green, v. 1, p. 363-372, 2007.

ALBERTON, C. L.; BECKER, M. E.; PINTO, S. S.; OLKOSKI, M. M.; KRUEL, L. F. M. Comparação dos efeitos de dois exercícios de hidroginástica nas respostas cardiorrespiratórias e na sensação subjetiva de esforço entre mulheres pósmenopáusicas e mulheres jovens In: Anais do XII Congresso de Ciências do Desporto e Educação Física dos Países de Língua Portuguesa. Porto Alegre, 2008.

ALBERTON, C. L.; TARTARUGA, M. P.; PINTO, S. S.; CADORE, E. L.; SILVA, E. M.; KRUEL, L. F. M. Cardiorespiratory responses to stationary running at different cadences in water and on land. The Journal of Sports Medicine and Physical Fitness, Torino, v. 49, p. 142-151, 2009.

ALBERTON, C. L.; KRUEL, L. F. M. Influência da imersão nas respostas cardiorrespiratórias em repouso. Revista Brasileira de Medicina do Esporte, São Paulo, v. 15, n. 3, p. 228-232, 2009.

ALBERTON, C. L.; ANTUNES, A. H.; PINTO, S. S.; TARTARUGA, M. P.; SILVA, E. M.; CADORE, E. L.; KRUEL, L. F. M. Correlation between rating of perceived exertion and physiological variables during the execution of stationary running in water at different cadences. Journal of Strength and Conditioning Research, Champaign, v. 25, n. 1, p. 155-162, 2011.

ALEXANDER, R. Mechanics and energetics of animal locomotion. In: Alexander R, Goldspink G, editors. Swimming. London: Chapman \& Hall, 1977, p. 222-248.

BARBOSA, T. M.; GARRIDO, M. F.; BRAGADA, J. Physiological adaptations to head-out aquatic exercises with different levels of body immersion. Journal of Strength and Conditioning Research, Champaign, v. 21, n. 4, p. 1255-1259, 2007.

BORG, G. Interindividual scaling and perception of muscular force. Kungliga Fysiografiska

Sällskapets I Lund Förhandlingar, Lund, v. 31, p. 117-125, 1961.

BORG, G. Psychophysical bases of perceived exertion. Medicine and Science in Sports and Exercise, Madison, v. 14, p. 377-381, 1982.

BORG, G. Escalas de Borg para a dor e o esforço percebido. São Paulo: Manole, 2000. 
CALEGARI-JACQUES, S. Bioestatística: Princípios e Aplicações. Porto Alegre: Artmed, 2004.

CASSADY, S. L.; NIELSEN, D. H. Cardiorespiratory responses of healthy subjects to calisthenics performed on land versus in water. Physical Therapy. Albany, v. 75, p. 532-538, 1992.

COOKE, C. B. Metabolic rate and energy balance. In: ESTON, R.; REILLY, T. Kinanthropometry and exercise physiology laboratory manual. London: E \& FN Spon, 1996. p. 175-195.

COSTA, G.; AFONSO, S.; BRAGADA, J. A.; REIS, V. M.; BARBOSA, T. M. Estudo comparativo das adaptações fisiológicas agudas durante a execução de três variantes de um exercício básico de hidroginástica. Revista Brasileira de Cineantropometria e

Desempenho Humano, Florianópolis, v. 10, n. 4, p. 323-329, 2008.

DARBY, L. A.; YAEKLE, B. C. Physiological responses during two types of exercise performed on land and in the water. The Journal of Sports

Medicine and Physical Fitness, Torino, v. 40, n. 4, p. 303-311, 2000.

DUNBAR, C. C.; ROBERTSON, R. J.; BAUN, R.; BLANDIN, M. F.; METZ, K.; BURDETT, R.; GOSS, F. L. The validity of regulating exercise intensity by ratings of perceived exertion. Medicine and Science in Sports and Exercise, Madison, v. 24, n. 1, p. 94-99, 1992.

GLEIM, G. W.; NICHOLAS, J. A. Metabolic costs and heart rate responses to treadmill walking in water at different depths and temperatures. American Journal of Sports Medicine, Baltimore, v. 17, n. 2, p. 248-252, 1989.

HALL, J.; MCDONALD, I. A.; MADDISON, P. J.; O'HARE, J. P. Cardiorespiratory responses to underwater treadmill walking in healthy females. European Journal of Applied Physiology, Berlin, v. 77, p. 278-284, 1998.

HOWLEY, E. T.; BASSET Jr., D. R.; WELCH, H. G. Criteria for maximal oxygen uptake: review and commentary. Medicine and Science in Sports and Exercise, Madison, v. 27, p. 1292-1301, 1995.

JOHNSON, B. L.; STROMME, S. B.; ADAMCZYK, J. W.; TENNOE, K. O. Comparison of oxygen uptake and heart rate during exercises on land and in water. Physical Therapy. Albany, v. 57, n. 3, p. 273-278, 1977.
KRUEL, L. F. M. Alterações fisiológicas e biomecânicas em indivíduos praticando exercícios de hidroginástica dentro e fora d'água. Tese de Doutorado. Universidade Federal de Santa Maria. 2000.

PANTOJA, P. D.; VENDRUSCULO, A. P; FAYH, A. P.; ALBERTON, C. L.; KRUEL, L. F. M. Respostas hemodinâmicas, cardiorrespiratórias e ocorrência de lesão muscular no meio aquático e terrestre em mulher não ativa: estudo de caso. Motriz. Revista de Educação Física. UNESP, Rio Claro, v. 12, n. 3, p. 277-282, 2006.

PINTO, S. S.; ALBERTON, C. L.; BECKER, M. E.; OLKOSKI, M. M.; KRUEL L. F. M. Respostas cardiorrespiratórias em exercícios de hidroginástica executados com e sem o uso de equipamento resistivo. Revista Portuguesa de Ciências do Desporto, Porto, v. 6, p. 336-41, 2006.

PINTO, S. S.; ALBERTON, C. L.; FIGUEIREDO, P. A. P.; TIGGEMANN, C. L.; KRUEL, L. F. M. Respostas de Freqüência cardíaca, consumo de oxigênio e sensação subjetiva ao esforço em um exercício de hidroginástica executado por mulheres em diferentes situações com e sem o equipamento Aquafins $\AA^{\circledR}$. Revista Brasileira de Medicina do Esporte, São Paulo, v. 14, n.4, p. 357361, 2008.

POHL, M. B.; McNAUGHTON, L. R. The physiological responses to running and walking in water at different depths. Research in Sports Medicine, Philadelphia, v. 11, p. 63-78, 2003.

RAFFAELLI, C.; LANZA, M.; ZANOLLA, L.; ZAMPARO, $P$. Exercise intensity of head-out water-based activities (water fitness). European Journal of Applied Physiology, Berlin, v. 109, p. 829-838, 2010.

SHONO, T.; FUJISHIMA, K.; HOTTA, N.; OGAKI, T.; UEDA, T.; OTOKI, K.; TERAMOTO, K.; SHIMIZU, T. Physiological responses and RPE during underwater treadmill walking in women of middle and advanced age. Journal of Physiological Anthropology and Applied Human Science. Chiba, v. 19, p. 195-200, 2000.

SVEDENHAG, J.; SEGER, J. Running on land and in water: comparative exercise physiology. Medicine and Science in Sports and Exercise, Madison, v. 24, n. 10, p. 1155-1160, 1992.

TIGGEMANN, C. L.; ALBERTON, C. L.; POSSER, M. S.; BRIDI, J.; KRUEL, L. F. M. Comparação das variáveis cardiorrespiratórias máximas entre a corrida em piscina funda e a corrida em esteira. 
Motriz. Revista de Educação Física. UNESP,

Rio Claro, v. 13, n. 4, p. 266-272, 2007.

Agradecimento: Os autores agradecem pelo apoio e incentivo dos órgãos CNPQ e CAPES.

Endereço:

Cristine Lima Alberton.

GPAT - Escola de Educação Física da UFRGS

Rua Felizardo,750

Porto Alegre RS Brasil

90690-200

Telefone: (51) 3308.5820

e-mail: tinialberton@yahoo.com.br

Recebido em: 6 de outubro de 2010.

Aceito em: 13 de abril de 2012.

\section{(c) (7)}

Motriz. Revista de Educação Física. UNESP, Rio Claro,

SP, Brasil - elSSN: 1980-6574 - está licenciada sob

Creative Commons - Atribuição 3.0 